\title{
Electrically Small U-slot Loaded Circular Microstrip Patch Antenna with CSRR
}

\author{
Mukul Chauhan \\ National Institute of Technical \\ Teacher's Training \& Research, \\ Chandigarh, India
}

\author{
Swapna Devi, PhD. \\ National Institute of Technical \\ Teacher's Training \& Research, \\ Chandigarh, India
}

\author{
Pradyot Kala, PhD. \\ Shree Ganpati Institute of \\ Technology, Ghaziabad, \\ U.P., India
}

\begin{abstract}
This paper presents the electrically small U-slot loaded circular microstrip patch antenna with Complementary Split Ring Resonator (CSRR). The technique of U slot loading is used for operating antenna in dual band and CSRR technique to make antenna compact and CSRR for compactness is used. The simple circular patch antenna resonates at $5.71 \mathrm{GHz}$ with return loss of $-11.38 \mathrm{~dB}$. The proposed antenna (with U-slot and CSRR) enhanced the matching upto- $37.47 \mathrm{~dB}$ at the 3.47 $\mathrm{GHz}$ and $-13.7 \mathrm{~dB}$ at the frequency $3.98 \mathrm{GHz}$ circular patch antenna and U-slot loaded circular microstrip patch antenna with CSRR are simulated using Ansoft HFSS and verified with the experimental results.
\end{abstract}

\section{General Terms}

Return Loss, Bandwidth, Antenna Size

\section{Keywords}

U-Slot loading, Complementary Split Ring Resonator (CSRR), Split Ring Resonator (SRR).

\section{INTRODUCTION}

Where the high performance with reduced size and cost required with, the microstrip antenna has the great advantage. These antennas are low profile, easily used with planar and non-planar surfaces, simple and low cost due to manufactured with printed circuit technology. In spite of various advantages, microstrip antennas have some disadvantages like low efficiency, low power handling capacity etc [1].

Different patch shapes are use in the microstrip antenna e.g. rectangular patch and circular patch etc. It is observed that a circular patch is more compact than a conventional rectangular patch antenna. [2]. Researchers focused on the compactness and achieve different techniques like slot loading, superstrate techniques etc. U-slot on patch provides the compactness bandwidth enhancement and dual-band operation [3].

Recently researchers are working on the metamaterial to increase compactness of the antennas. Metamaterial is the material which does not exist in the reality that realized in artificial manner which gives the electromagnetic confinement. Metamaterial is classified as negative index materials and single index materials [4]. In negative index materials both permittivity and permeability are negative. In single index metamaterial, only one of the permittivity and permeability is negative both are not the negative at the same time, this is categories as the ENG (Epsilon negative media) permittivity is negative and permeability is positive, On the other hand in MNG (Mu-negative media) permeability is negative and permittivity is positive. The first structure is given by Shelby Smith and Schultz that has been used to prove the existing of metamaterial was a split ring structure invented in 2001 at the University of California [5]. The use of the metamaterial is to improve some basic antenna features (impedance matching, gain, bandwidth, efficiency, front-toback ratio, etc.). Metamaterial technique is the novel way to reduce the size of the antenna in respect to the existing size reduction techniques of antenna [6].

Initially the existence of original medium was proposed in [7] consists of a bulky combination of metal wires and split-ring resonators (SRRs). SRR are the planar structures and can be replaced by planar strips [8]. The electromagnetic properties of SRRs have been showed in [9]. The equivalent circuit for the SRR and CSRR are shown in Fig.1, where C is the total capacitance between the rings of SRR. SRR can be taken as the resonant dipole which may be excited by an axial magnetic field [10]. This concept is extended to the CSRR in which the resonant magnetic polarization ability along its yaxis [11]. The resonant frequency of circular patch for the dominant mode is defined by the equation (1)

(a


(b
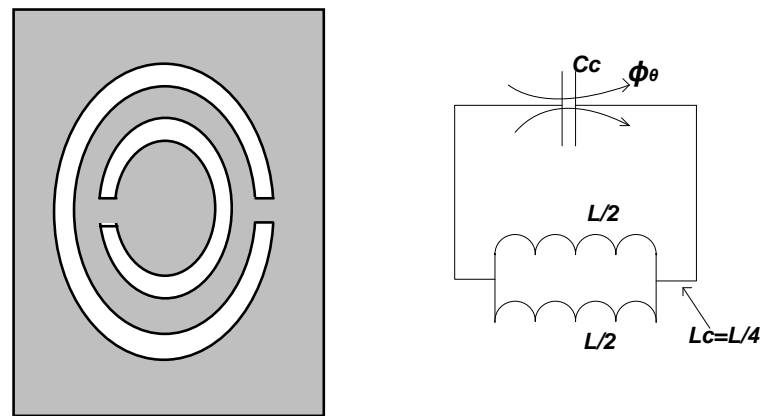

Fig. 1. Topologies of the: (a) SRR and (b) CSRR, and their equivalent-circuit Grey zones represent the metallization [10]. 


$$
f=\frac{1.8412 \times 3 \times 10^{11}}{2 \times 3.14 \times \sqrt{\varepsilon_{r}} \times a}
$$

Where $a$ is the radius of the circular patch

In proposed antenna the size of the antenna is reduced by use of CSRR technique and dual band operation is performed with the use of U-slot loading on circular patch. The effect of the rotation of cuts on CSRR is shown in comparisons of results. The concept of frequency shifting towards the lower value is presented with the use of CSRR due to the characteristics of the metamaterial.

\section{ANTENNA DESIGN}

Figure 2 depicts the geometrical structure of circular U-slot loading with its dimension notations. Figure 3 shows the geometrical view of the CSRR on the ground of the proposed antenna with its notations.

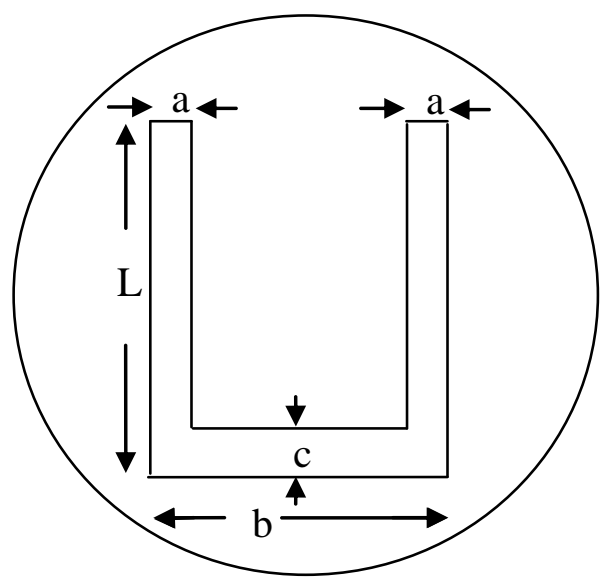

Fig 2: Sketch and geometrical structure of patch view of U-slot loaded electrically circular microstrip patch

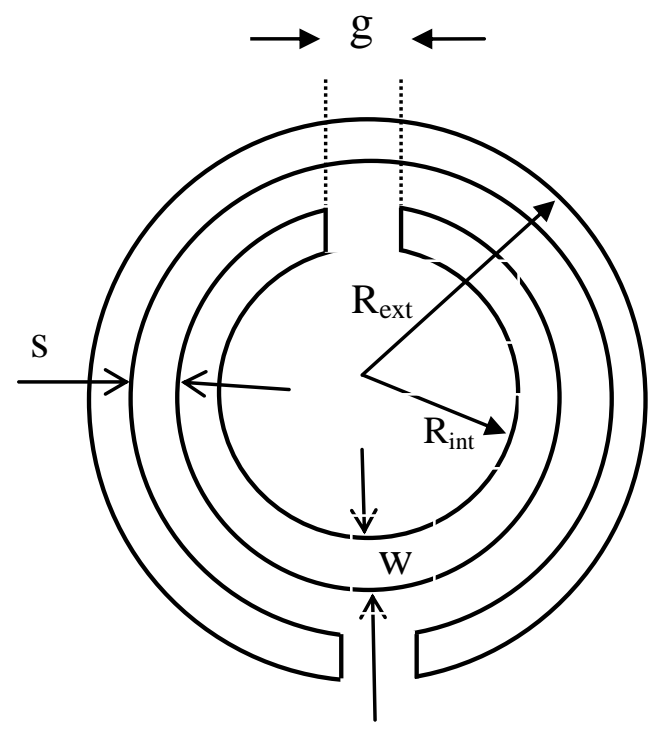

Fig 3: Sketch and geometrical structure of ground view of circular microstrip patch antenna with CSRR.
Table 1 shows the dimensions of the proposed antenna and the cuts in CSRR on the ground are aligned in $\mathrm{x}$ - direction.

Table.1 Design Parameters of proposed antenna design

\begin{tabular}{|c|c|}
\hline Substrate material-Fr4 & $\varepsilon_{r}=4.4$ \\
\hline $\begin{array}{c}\text { thickness of dielectric } \\
\text { substrate }\end{array}$ & $h=1.6 \mathrm{~mm}$ \\
\hline radius of circular patch & $r=6.88 \mathrm{~mm}$ \\
\hline $\begin{array}{c}\text { length \& width of rectangular } \\
\text { slots }\end{array}$ & $\begin{array}{c}L=8 \mathrm{~mm}, b=8 \mathrm{~mm}, c=1 \\
\mathrm{~mm}, a=1 \mathrm{~mm}\end{array}$ \\
\hline $\begin{array}{c}\text { radius of the CSRR on the } \\
\text { ground }\end{array}$ & $\begin{array}{c}R_{\text {ext }}=5.8 \mathrm{~mm}, R_{\text {int }}=3.1 \mathrm{~mm} \\
s=\mathrm{w}=1 \mathrm{~mm} \\
g=1.5 \mathrm{~mm}\end{array}$ \\
& $\begin{array}{c} \\
\end{array}$ \\
\hline
\end{tabular}

The physical dimensions are evaluated and put in the Ansoft HFSS as a 3D EM field solver to reach the final real structure.

\section{RESULTS AND DISCUSSION}

In this section simulated results and experimental results of the proposed antennas are discussed.

\subsection{Simulated Results}

Initially the circular patch antenna without any changes is simulated and the resonant frequency is $5.7 \mathrm{GHz}$ with the return loss of $-11.97 \mathrm{~dB}$ simulated result shown in figure 3 .

Now the first proposed U-slot loaded circular microstrip patch antenna with CSRR having the cuts in $\mathrm{x}$ direction is designed and simulated. The proposed antenna shows the resonant frequencies at $3.47 \mathrm{GHz}$ and $3.98 \mathrm{GHz}$ with the return loss of $37.4 \mathrm{~dB}$ and $-13.7 \mathrm{~dB}$ respectively, simulated results shown in figure 4.

Table.2 Comparison in reduction of antenna size

\begin{tabular}{|c|c|c|c|}
\hline $\begin{array}{l}\text { Frequency } \\
\text { (GHZ) }\end{array}$ & $\begin{array}{c}\text { Area of } \\
\text { the } \\
\text { circular } \\
\text { patch } \\
\left(\mathbf{m m}^{\mathbf{2}}\right)\end{array}$ & $\begin{array}{c}\text { Area of } \\
\text { proposed } \\
\text { antenna } \\
\left(\mathbf{m m}^{\mathbf{2}}\right)\end{array}$ & $\begin{array}{c}\text { \% reduction } \\
\text { in area }\end{array}$ \\
\hline 6 & 153.84 & - & - \\
\hline 5 & 220.72 & - & - \\
\hline 3.98 & 348.63 & 153.84 & 65.1 \\
\hline 3.4 & 477.39 & 153.84 & 67.7 \\
\hline
\end{tabular}

It is clear from the results that antenna become dual band which prove the significance of the U- slot loading and the reduction of size or in other terms the shifting the resonant frequency from towards the lower frequency is shown in the table 2. The effect of the rotation in the CSRR structure on ground is shown by the variation in CSRR cuts. In [12] variation of CSRR positions are used using rectangular slots. 


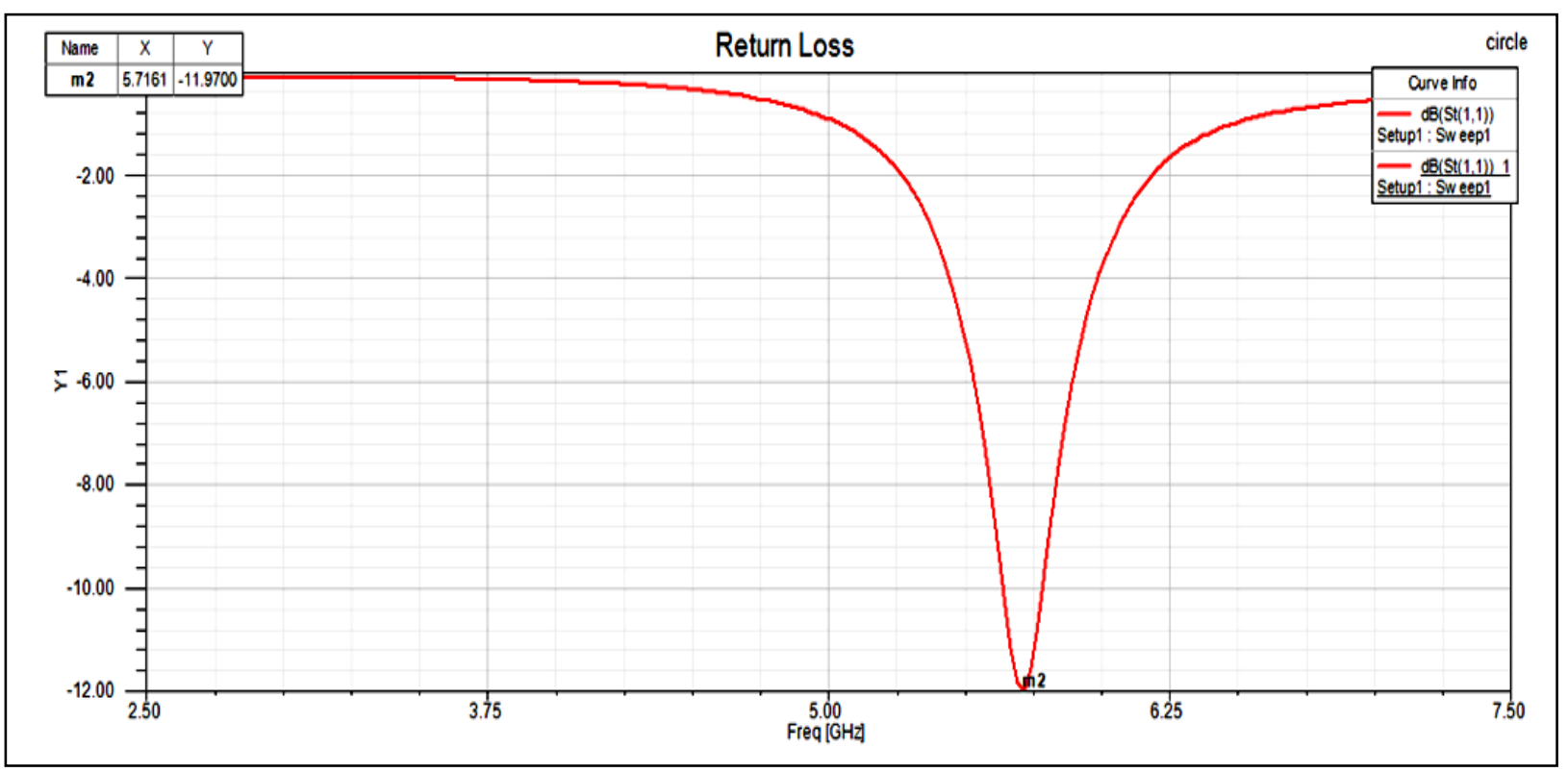

Fig 4: Reflection Coefficient of the Circular microstrip patch antenna

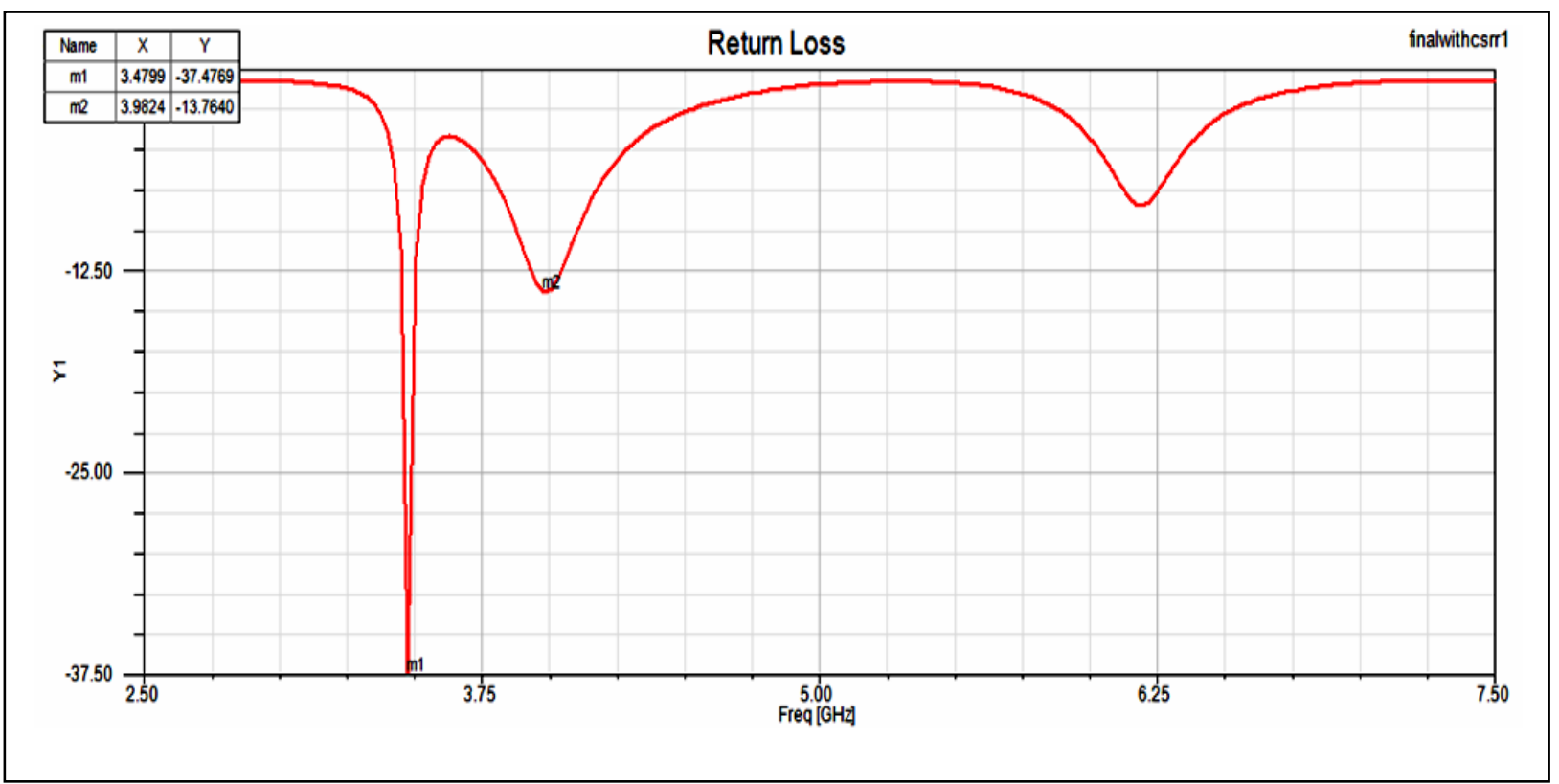

Fig 5: Reflection Coefficient of the proposed U-slot loaded Circular microstrip patch antenna with CSRR (cuts in $x$ direction)

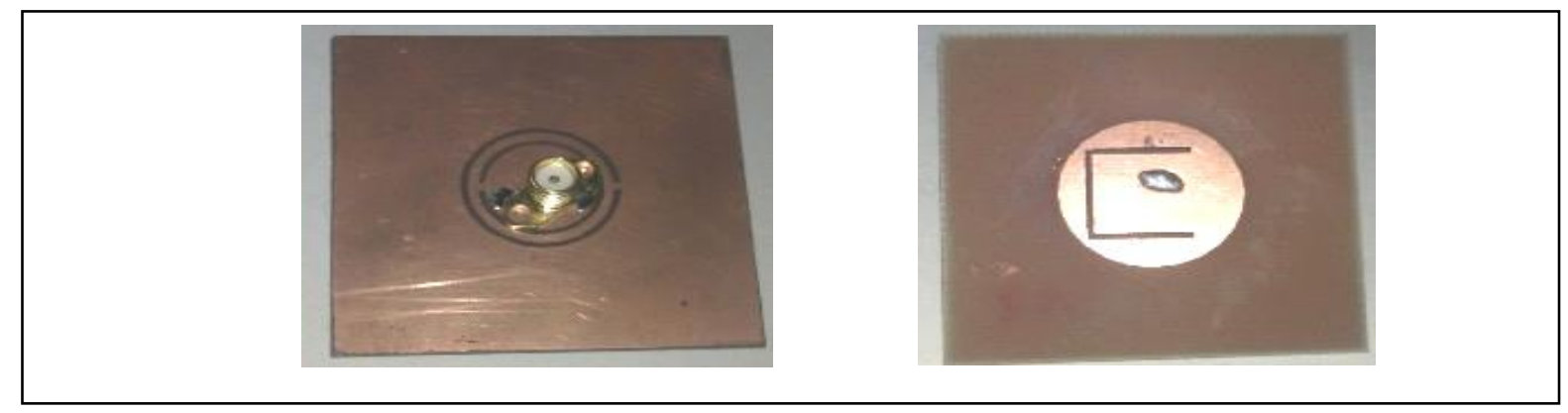

Fig 6: Ground view and top view of fabricated antenna 


\subsection{Experimental results}

The proposed antenna is fabricated on the FR4 with the substrate thickness of $h=1.6 \mathrm{~mm}$. The antenna is tested on the Vector Network analyzer. Figure 6 shows the top view and the ground view of the proposed antenna. Results obtained by fabricated antenna are approximately equal to the simulated results by the Ansoft HFSS.

\section{CONCLUSION}

A minimized design of antenna is presented with the use of $U$ slot loading and use of CSRR techniques with improvement in return loss $\left(S_{11}\right)$ with the frequency shifting to lower value. The size of the antenna is reduced up to $67 \%$. The properties of the antenna may easily changed by varying the width of the U slot, width and radius of the CSRR and spacing between the rings of CSRR. Simulated results of the proposed antenna are verified with experimental results. Proposed antenna can be used in many dual band wireless communications.

\section{REFRENCES}

[1] C. A. Balanis, Modern Antenna handbook, Hoboken, Wiley \& Sons, 2008.

[2] M. D. Deshpande, "Analysis of stub loaded microstrip patch antennas", IEEE International Symposium on Antennas and Propagation Society, vol. 2, pp. 610 - 613, 1997.

[3] K. F. Lee, K. M. Luk, K. F. Tong, S. M. Shum, T. Huynh, and R. Q. Lee, "Experimental and simulation studies of the co-axially fed U-slot rectangular patch antenna”, IEE Proc Microwave Antenna Propagation, pp. 354-358, 1997.

[4] T. J. Cui, R. Liu, B. Zhao, X. Q. Lin and H. F. Ma, "A New Metamaterial Structure to Amplify Evanescent Waves and Its Applications in Microwave Components," in Proc. Int. Workshop on Antenna Technology, IWAT 2007, pp. 527-527, 2007.

[5] Engheta, Nader; Richard W. Ziolkowski. Metamaterials: Physics and Engineering Explorations. Wiley \& Sons. pp. xv, 3-30, 2006.

[6] J.S Lim, C.B. Kim, J.S. Jang, "Design of a subwavelength patch antenna using metamaterials", Microwave Conference, EuMC 2008, 38th European, pp. 547-550, Amsterdam, 2008.

[7] R. Smith, W. J. Padilla, D. C. Vier, S. C. Nemat-Nasser, and S. Schultz, "Composite medium with simultaneously negative permeability and permittivity", Phys. Rev. Letter, vol. 84, pp. 4184-4187, 2000.

[8] J. B. Pendry, A. J. Holden, D. J. Robbins, and W. J. Stewart, "Magnetism from conductors and enhanced nonlinear phenomena", IEEE Trans. Microw. Theory Tech., vol. 47, pp. 2075-2084, 1999.

[9] R. Marqués, F. Mesa, J. Martel, and F. Medina, "Comparative analysis of edge- and broadside-coupled split ring resonators for metamaterial design-Theory and experiment", IEEE Trans. Antennas Propag., vol. 51, pp. 2572-2581, 2003.
[10] R. Marqués, F. Medina, and R. Rafii-El-Idrissi, "Role of bianisotropy in negative permeability and left handed metamaterials", Phys. Rev. B. Condens. Matter, vol. 65, pp. 144 441-144 441, 2002.

[11] Ansari, J. A. and R. B. Ram, "Analysis of broad band Uslot microstrip patch antenna," Microwa. Opt. Tech. Letter., Vol. 50, No. 4, 1069-1073, 2008.

[12] Dong, Y ; Toyao, H. ; Itoh ,T. ," Design and Characterization of Miniaturized Patch Antennas Loaded With Complementary Split-Ring Resonators", IEEE Transaction on antenna \& propagation, vol. 60 , pp-772785, 2012.

[13] Bahl, J. and P. Bhartia, Microstrip Antennas, Artech House, Dedham, 1980.

[14] Tamma, V.A.; Tin-Ei Wang; Kuester, E.F.; Wounjhang Park; Holloway, C.L.; Ji Zhou, "Metamaterial inspired electrically small patch antenna", In Proc. IEEEAntennas and Propagation, pp.775-778, 2011.

[15] S.S Pattnaik, J.G Joshi,. S Devi, M.R. Lohokare, "Electrically Small rectangular microstrip patch antenna loaded with metamaterial", In Proc. IEEE Antennas Propagation and EM Theory, pp. 247-250, 2010.

[16] Bilotti, F.; Alu, A.; Vegni, L., "Design of Miniaturized Metamaterial Patch Antennas With $\mu$-Negative Loading”, IEEE Trans. on Antennas and Propagation, vol. 56, pp.1640-1647, 2008.

[17] Li B., B. Wu, and C.-H. Liang, "Study on hign gain circular waveguide array antenna with metamaterial structure," Progress In Electromagnetics Research, PIER 60, 207-219, 2006.

[18] R.W. Ziolkowski, Lin Chia-Ching, "Metamaterialinspired magnetic-based UHF and VHF antennas" In Proc. IEEE Antennas and Propagation Society International Symposium, pp.1-4, 2008.

[19] J.G. Joshi, S.S Pattnaik,, S. Devi, M.R Lohokare, C. Vidyasagar, "Offset fed diamond shaped split ring (DSSR) planar metamaterial antenna," IEEE Conference on Applied Electromagnetics, pp.1-4, 2009.

[20] Lee Cheng-Jung, K.M.K.H Leong, T. Itoh, "Compact Dual-Band Antenna Using an Anisotropic Metamaterial," In Proc. IEEE Microwave Conference, pp. 1044-1047, 2006.

[21] P. Jin and R. W. Ziolkowski, "Low Q, electrically small, efficient near field resonant parasitic antennas", IEEE Transaction on Antennas Propagation, vol. 57, pp. 25482563, 2009.

[22] James S. McLean, "A Re-Examination of the fundamental limits on the radiation Q of electrically small antennas", IEEE Transactions on Antennas and Propagation, vol. 44, No. 5, pp. 672-676, 1996. 